\title{
Repair Effect of Mesenchymal Stem Cells on Fracture Model Animals
}

\author{
LIU Jia ${ }^{1}$, ZHOU Peng ${ }^{1}$, LIU Xiaoyun ${ }^{2}$ \\ ${ }^{1}$ Department of Basic Medicine, ChangSha Medical University, Changsha, 410219, China \\ ${ }^{2}$ The Affiliated Hospital of ChangSha Medical University, Changsha, 410219, China
}

Key words: Mesenchymal Stem Cells; SDF-1; Homing of stem cells

\begin{abstract}
In this paper, we propose the role of mesenchymal stem cells in fracture repair. We investigated the role of bone marrow mesenchymal stem cells in the healing process of fracture and the effect on the migration of endogenous bone marrow mesenchymal stem cells. Experiments show that exogenous bone marrow mesenchymal stem cells implanted in femur fractures in vivo can make endogenous bone marrow mesenchymal stem cells in vitro migration ability and fracture site of nerve growth factor expression increased, which promotes rat fracture healing.
\end{abstract}

\section{Introduction}

Fracture is a more common orthopedic disease. It often causes disorders of the patient's joints and leads to a series of complications. It brings great harm to the patient's physical and mental. Promoting the normal healing of fractures is particularly important [1]. Those who can not heal the fracture without further intervention are called bone nonunion. Bone defect and nonunion are common in patients with advanced complications of fracture. The incidence is 5-10\%. Fractures cause pain and burden to patients, families and society. There have a great impact on the patient's physical and mental health. Therefore, the mechanism of bone healing is of great economic and social significance [2].

Fracture healing is an extremely complex and continuous process. Generally it is divided into inflammatory response period, repair period and plastic period. The three stages are intertwined with each other [3]. Fracture healing requires the processes of hematoma formation, fibrous callus formation, bone callus formation and callus remodeling. Finally it reaches the perfect healing. Eventually bones are normal in structure and function. The initial stage of the fracture is the hematoma formation. In the bone tissue rupture, the blood flow into the end of the formation to form a hematoma. Macrophages enter the fracture area to clean up. It prepares for repair. But there will be mild neutrophil infiltration, accompanied by mild sexual response. This is not conducive to fracture healing [4].

Delayed healing of the fracture can lead to severe difficult to heal dysfunction. Mesenchymal stem cells (MSCs) are widely distributed in the tissues of bone marrow, fat, periosteum, muscle, synovium, tendon, umbilical cord blood, pulp, peripheral blood vessels. The isolated mesenchymal stem cells can be separated from these tissues with multiple differentiation potential, with high self-renewal ability and proliferation ability [5]. It can differentiate into bone, cartilage, fat, muscle, tendon, vascular endothelial cells and nerve cells under different induction and differentiation conditions. Bone marrow mesenchymal stem cells are readily available. It can be amplified in vitro fast. It`s immune rejection is small. It can be long-term living in the host body, which still has differentiation function after multiple passages and is easy to transfection. Whether in local or systemic injection, or in tissue engineering, mesenchymal stem cells in bone and cartilage injury repair have played an indispensable role. It has a good application prospects.

In recent years, with the development of stem cell culture, separation and other technology to make the treatment of nonunion of fracture have more choices. In this paper, bone marrow mesenchymal stem cells were injected into the fractured rats to explore the effect of bone marrow 
mesenchymal stem cells. In the case of exogenous bone marrow mesenchymal stem cells, the migration ability of bone marrow mesenchymal stem cells and the expression of nerve growth factor in the fracture femur of the femur were increased, which could affect the fracture healing.

This section is as follows. Chapter 2 describes the origin of mesenchymal stem cells. Chapter 3 shows the homing process of mesenchymal stem cells in fracture repair. Chapter 4 describes the optimal time of exogenous injection of bone marrow mesenchymal stem cells homing to promote fracture healing.

\section{The Sources of Mesenchymal stem cells}

Mesenchymal stem cells are a special stem cell. It has the ability to multi-directional differentiation. Mesenchymal stem cells can be transformed into osteocytes, chondrocytes, adipocytes, fibroblasts and muscle cells in cytokines and various microenvironments. Mesenchymal stem cells are widely distributed. Many organizations have found its presence, such as bone marrow, adipose tissue, muscle tissue and peripheral blood. Bone marrow - derived mesenchymal stem cells proliferate strongly. The study found that it can still maintain its multi-differentiation potential while it was expanded. Bone marrow mesenchymal stem cells are readily available, which can be amplified in vitro fast, immune rejection is small, and can be long-term living in the host body. It has differentiation function after multiple passages and is easy to transfection and so on. BMSC is easy to escape the immune surveillance of the body, with very low immunogenicity. Therefore, it only occurs mild immune rejection in allogeneic transplantation. This provides the possibility of long-term coexistence in the host. In the field of orthopedics, BMSCs are ideal seed cells for bone and cartilage tissue engineering. It also plays an important role in gene therapy. Figure 1 shows the sources of mesenchymal stem cells.

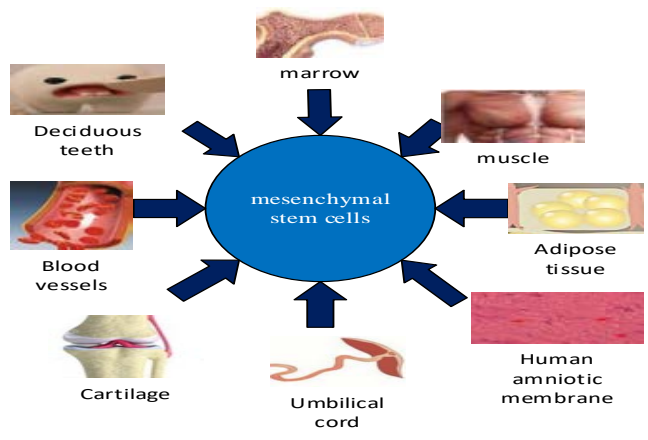

Figure 1. The sources of mesenchymal stem cells

At present, mesenchymal stem cells are isolated and cultured by density gradient centrifugation, adherence screening and immunoassay. Density gradient centrifugation and adherence screening method are simple and easy. Although the separation of the obtained cell purity is low, it has a good cell differentiation ability. Immunoassay is based on BMSC surface antigen markers and is sorted using the corresponding fluorescein-labeled flow cytometry. Immune magnetic bead sorting method is combined with a specific single cast of magnetic beads combined. The high purity of the cells obtained is high, but the price is expensive, which makes its application subject to certain restrictions.

\section{Homing of Mesenchymal Stem Cells in Fracture Repair}

Under the influence of certain signals, mesenchymal stem cells have the ability to aggregate and migrate to certain specific tissues, which is called cell homing. Stem cell homing includes the 
following four consecutive processes. The four processes are cell recruitment, migration, proliferation, differentiation. The study found that the body after multiple tissue damage, such as the heart, liver, brain, nerve, kidney, epithelial and other organizations, has the processes of mesenchymal stem cells homing and promotes the repair of injury.

Fractures have also confirmed that adjacent and systemic osteogenic stem cells are recruited and migrated to fractures, which promotes the repair of fractures. Fractures in the periosteum and adjacent BMSCs is very limited. Bone repair process using tissue engineering bone, stents and other bridges, also needs to gather in the fracture of a sufficient number of BMSCs. So it is extremely important to mobilize mesenchymal stem cells throughout the body to the fracture. Stem cell homing must be the result of the interaction of a factor secreted by the fracture with the corresponding receptor on the surface of the stem cell. The pathway of stromal cell-derived factor-1 (SDF-1) and its receptor (CXCR4) has been shown to play an important role in the repair of heart, liver, kidney and nerve. This pathway also plays an important role in fracture repair. The expression of CXCR4 on the surface of BMSCs secreted by SDF-1 and BMSCs is affected by the gradient of SDF-1. Stem cells migrate to bone lesions. Figure2 shows the process of homing.

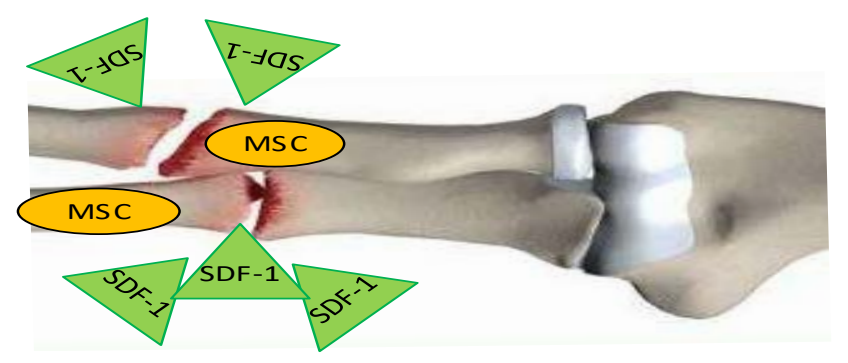

Figure 2. Mesenchymal stem cells induced homing to bone injury

MSCs plays an important role in multiple damage repair. In the comparison of many mesenchymal stem cells, bone marrow mesenchymal stem cells is the most effective. Bone marrow mesenchymal stem cells (BM-MSCs) are subgroups of stromal cells. It is located on the intima of the bone marrow cavity. Bone marrow is still an important source of access to mesenchymal stem cells because of its good access and a relatively high concentration in the bone marrow.

Stem cells have limited origin. Only $0.001 \%$ to $0.01 \%$ of the mononuclear cells in the bone marrow are mesenchymal stem cells. Therefore, an effective method of isolation and culture is necessary. When the damage is large, their own mesenchymal stem cells are often inadequate. Vitro mesenchymal stem cells in vitro expansion and culture back to the body after the method is more commonly used. And bone marrow mesenchymal stem cells are still the most widely used and most readily available, leading to the strongest role of bone marrow mesenchymal stem cells.

\section{The optimal time of exogenous injection of bone marrow mesenchymal stem cells homing to}

\section{promote fracture healing}

Bone marrow mesenchymal stem cells (BMSCs) can promote fracture healing. The optimal injection time point for BMSCs is uncertain. In this study, BMSCs were injected into the tail vein at the first week, the second week and the third week after the mice were closed by fracture. It was suggested that BMSCs were the best time to promote fracture healing. The test is to investigate the 
role of BMSCs in promoting SDF-1 / CXCR4 pathway during fracture healing, and to explore the possible mechanism of the best time point.

The mice were divided into three groups according to the intervention time of MSCs injection. Experimental grouping is as shown in Table 1.

Table 1 Experimental grouping

\begin{tabular}{|l|l|l|}
\hline Group No. & $\begin{array}{l}\text { Injection } \\
\text { time(week) }\end{array}$ & The number of rats \\
\hline 1 & 1 & 50 \\
\hline 2 & 2 & 50 \\
\hline 3 & 3 & 50 \\
\hline
\end{tabular}

After 4 weeks, we can get the results of figure 3 .

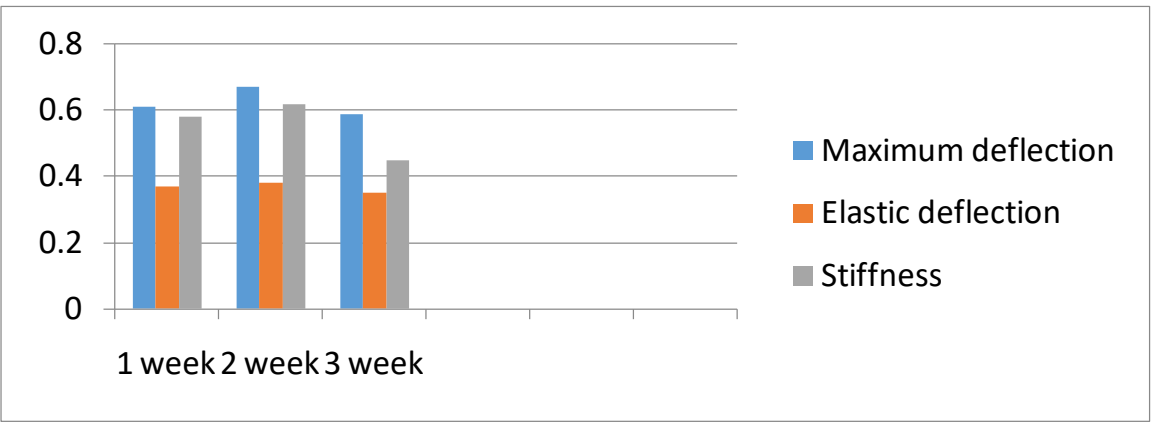

Figure 3. Mesenchymal stem cells induced homing to bone injury

It can be seen from the results, the first week and second week after injection of BMSCs can be extended MSC homing and retention time. One of the best injections in the second week. The third week of injection is weak. The injection of BMSCs in the first and second weeks after fracture increased the mechanical properties of callus, which is better than the third week.

\section{Summary}

In the case of exogenous bone marrow mesenchymal stem cells, the migration ability of bone marrow mesenchymal stem cells and the expression of nerve growth factor in the fracture of the femur fracture were increased, which could affect the fracture healing of the rats. After fractures, the fractures produce a large amount of SDF-1. SDF-1 can react with BMSCs expressing CXCR4, and promote BMSCs to migrate to the bone. It can accelerate the healing of the fracture.

\section{Acknowledgement}

This work was supported by Hunan Provincial Health and Life Program Research Project (B2017042).

\section{References}

[1] Xie C, Yang Z, Suo Y, et al. Systemically Infused Mesenchymal Stem Cells Show Different Homing Profiles in Healthy and Tumor Mouse Models [J]. Stem Cells Translational Medicine, 2017.

[2] Tewari D, Khan M P, Sagar N, et al. Ovariectomized Rats with Established Osteopenia have Diminished Mesenchymal Stem Cells in the Bone Marrow and Impaired Homing, Osteoinduction and Bone Regeneration at the Fracture Site [J]. Stem Cell Reviews and Reports, 2015, 11(2):309-321.

[3] Yao W, Lay Y E, Kot A, et al. Improved Mobilization of Exogenous Mesenchymal Stem Cells to 
Bone for Fracture Healing and Sex Difference[J]. Stem Cells, 2016, 34(10):2587.

[4] Zhou Q I, Yang C, Yang P. The Promotional Effect of Mesenchymal Stem Cell Homing on Bone Tissue Regeneration [J]. Current Stem Cell Research \& Therapy, 2015, 10.

[5] Liang X, Huang X, Zhou Y, et al. Mechanical Stretching Promotes Skin Tissue Regeneration via Enhancing Mesenchymal Stem Cell Homing and Transdifferentiation [J]. Stem Cells Transl Med, 2016, 5(7):960.

[6] Stuermer E K, Lipenksy A, Thamm O, et al. The role of SDF-1 in homing of human adipose derived stem cells.[J]. Wound Repair \& Regeneration, 2015, 23(1):82-89.

[7] Wu M, Ji S, Xiao S, et al. JAM-A promotes wound healing by enhancing both homing and secretory activities of mesenchymal stem cells[J]. Clinical Science, 2015, 129(7):575-88.

[8] Naderi - Meshkin H, Matin M M, Heirani - Tabasi A, et al. Injectable hydrogel delivery plus preconditioning of mesenchymal stem cells: exploitation of SDF - 1/CXCR4 axis toward enhancing the efficacy of stem cells' homing[J]. Cell Biology International, 2015, 40(7):730.

[9] Ho C Y, Sanghani A, Hua J, et al. Mesenchymal stem cells with increased SDF-1 expression enhanced fracture healing.[J]. Tissue Engineering Part A, 2014. 